\title{
Application of P2P Streaming Media to Network Teaching
}

\author{
Li Jie \\ College of Foreign Languages, Hebei University, Baoding, Hebei 071000 \\ huner2011@foxmail.com
}

Keywords: P2P, Streaming media, English network video teaching

\begin{abstract}
Traditional network education is based on centralized $\mathrm{C} / \mathrm{S}$ or $\mathrm{B} / \mathrm{S}$ mode. With rapid increase of network education users, the video server is overloaded under centralized $\mathrm{C} / \mathrm{S}$ or $\mathrm{B} / \mathrm{S}$ mode, which is easy to make network bandwidth bottleneck and influences service quality. Therefore, establishing network education system based on P2P streaming media can improve the service quality of video teaching. The paper deeply studies the problems of applying P2P streaming media model in network video teaching in English teaching, and proposes implementation idea, method and system structure.
\end{abstract}

\section{Application of P2P Network to Network Education}

P2P(Peer To Peer) means that the nodes in distributed system is logical and equivalent. Compared with traditional C/S architecture model, P2P architecture model doesn't differentiate server and client, and the nodes in the system can make direct data communication without needing the transference of server nodes. After applying the architecture, each node not only is the user of the service, but also can be the provider of service, which can fully use processing capacity of terminal device, and makes low-cost and distributed service possible. The autonomy, cross-platform and unified service and access interface of each node in P2P network makes that P2P mode can be applied in wider range under traditional mode. The technique has been the research focus of internet application, and has been widely applied in many aspects of internet.

Rapid increase of network education users brings new problems for network teaching. Most network teaching platforms apply C/S mode. Under the mode, the requested server of each user responds alone and establishes specialized communication. The rapid increase of network users makes load of server increase, which makes large cost and administrative overhead. And all resources are put on the server under the mode, which makes it uneasy for resource communication of users. And the users can't get the latest teaching information, and the streaming media server can't provide service for the users needing service[1]. P2P network is a good way to solve the existing problems of network teaching platform. The idea of weakening central server in P2P network provides a solution for solving the heavy load of server in network teaching. The application of P2P streaming media can make network teaching platform serve for more users at the same time, which solves the limitation of processing ability of server in network platform.

\section{System Design Objective}

In traditional teaching mode, classroom is an important place for teachers to teach knowledge to students. Limited by classroom space and schooltime, teacher resources can't be fully used. If applying P2P streaming media technique to online class of network teaching can break the limitation of time and classroom space, more students can enjoy the class of excellent teachers [2]. According to the requirements of network teaching, online class supports lots of students at the same time, and the waiting time for students is short. Online class system has good recovery mechanism. After some students are lost, other students can be not influenced, or reenter online class as soon as possible. 


\section{System Design}

System structure. Online class in network teaching needs to use P2P network to broadcast the teaching process of teachers. And video devices are used to acquire video and audio data in teaching process, and the acquired data receives real-time coding by coders, and is transferred into video stream for broadcasting. The students access to the online class by client side for learning. Through P2P network, teachers can teach students anywhere, which can fully use teacher resources. Video stream data is transmitted in P2P network consisting of client sides, which reduces the load of server [3]. The structure of online class is shown in Fig. 1.

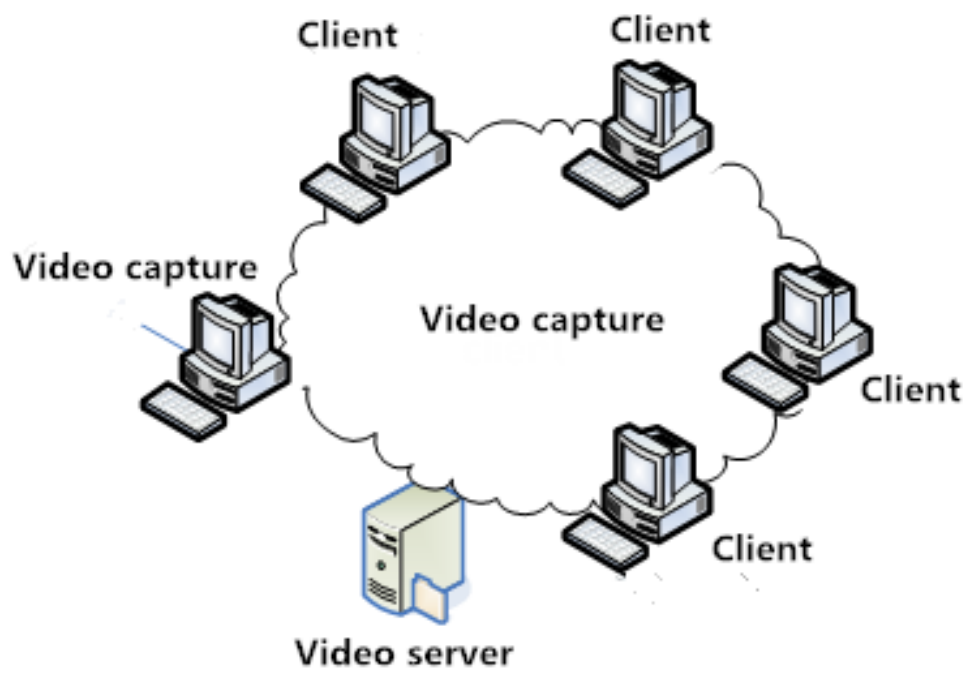

Fig. 1 Online class structure

System Module. According to the structure of online class system, online class can be divided into broadcasting server, Web server and client side. Broadcasting server acquires the events to be broadcast, and the events are coded into video stream. It consists of direct broadcast resource and data transmission module and creating information module. Creating information module establishes and broadcasts the information relating to the broadcast events. The direct broadcast resource consists of video and audio collector, video and audio coder and video stream memory [4]. Web server receives the broadcast information sent from direct broadcast resource, and displays with the form of page. Web server manages the node and data block information in P2P network. Web server consists of information management, resource management and data transmission module.

The function of information management module is to analyze the information sent by direct broadcast resource and show the analyzed information. Data transmission module sends and receives the data. Resource management records the nodes in network and counts the number of data blocks of each node. Client side consists of management, data transmission and broadcaster.

Management module selects the nodes and the data blocks to be loaded according to the requirements of users. Data transmission module downloads the data with relevant nodes according to the decision of management module. After downloading data, the broadcaster module broadcasts the data.

System flow. Direct broadcast resource server selects the events to be broadcast, acquires the video and audio of the broadcast events, encodes the acquired data, and converts the data into direct broadcast video stream. It broadcasts the video stream, receives and stores the broadcast video stream. Broadcast server establishes the information according to the broadcast events, and sends the information to Web server. After receiving the information, Web server analyzes the information, and shows the analyzed results.

The students log in Web server to find the broadcast resources to be learned, and download the information relating to resources. The local clients unfold the information of broadcasting resources, and download the resources in the nodes from Web server. According to the node selection 
algorithm and data selection algorithm, the nodes to be connected and the data blocks to be downloaded are determined. And the required data blocks are downloaded in the relevant nodes, and are broadcast by player after download.

\section{System Implementation}

Generation of live video stream. In the process of acquiring the original data and coding the data of video and audio, it is common to use Windows Mediaeom ponentsl developed by windows Cooperation is used for implementation. After installing video and audio acquisition device, the events to be acquired are selected [5]. The encoder of Windows Media is opened to establish a new session and select custom session.And it enters the setting interface to set the video and audio acquisition device according to requirements. And encoding begins after setting. And Windows Media Components encodes the acquired data, and video stream of Windows Media generates.

Reception of video stream. In the process of receiving video stream, the communication of broadcaster and Windows Media Components is simulated. And the pseudocode implementing the reception of video stream is as follows.

Reev_Media_Data(T)://T means disengaging request.

soeket(s);// establishing socket $\mathrm{S}$

$\mathrm{T}=1$;

while(T)

\{

while(1)

\{

send(play);// simulating broadcaster data and sends to the server $\quad$ recv(buf);//receiving the data from server

result=analysis(buf);//analyzing if the data which is received from server has "Http/1.0 $200 \mathrm{OK}$ $\left\lfloor n \backslash r^{\prime \prime}\right.$

if(result ==1);// it means the returning information of the received server is right.

break;

else;//it means that the received information is not right, and the request is sent to the server again.

\}

while(l)

\{

send(play);//simulating and sending the broadcaster data to the server

recv(buf);//receiving the data returning from the server result=analysis(buf);//analyzing if the received data from the server has "Http/1.0 200 OK $\backslash n \backslash r$ "

if(result $==0$ );//it means that the returning information of the received server is right.

break;

else

recv(buf);//video head

save(buf);// storing video head data

recv(buf);// receiving video data

save(buf);// storing video data

\}

\}

$\operatorname{lf}(\operatorname{recv}()) \mathrm{T}=0 / /$ if the request is received.

Crossing NAT. When the the nodes in private network communicate, the pseudocode crossing NAT is as follows.

$\mathrm{S}$ :// server

A:// a node in private network

$\mathrm{B}: / /$ the node in another private network 
socket(A);//establishing sock A for node A

socket(B);//establishing sock B for node B

A->send(S);//node A communicates with the server $S$

$\mathrm{B}->$ send(S);//node B communicates with the server $\mathrm{S}$

A-> send(S, B);//node A request to communicate with node B

S-> send(B, A);// server sends orders to B, and establishes a Session relating to node A on NAT.

B->send(A);// node B sends information to A and establishes a Session relating to node A on NAT.

B->send(S, A);//node B sends information to server, and Session of A on NAT has been established.

s->send(B, A);// server tells node A to communicate with node B.

A->send(B);// node A sends information to node B, and establishes a Session relating to be on NAT.

B->recv(A);//node $B$ receives the information sent by node $A$

$\mathrm{B}->$ send(A);//node $\mathrm{B}$ sends information to node $\mathrm{A}$

A->reev(B);//node A receives the information sent by node B

4.4 Sending resource information

After video live resource receives the inputted live information, the content is transferred into a XML file, and XML file is sent to Web server. And the content of XML file is as follows.

$<$ ?Xml version="1.0"? $>$

$<$ Resources $>$

$<$ url> http://219.245.31.22:1234

$</$ URL $>$

$<$ name $>$ match advance $</$ name $>$

$<$ description $>$ compulsory course $<$ /deseription $>$

$<$ Bitrate $>400</$ Bitrate $>$

$<$ Type $>$ wmv $</$ type $>$

$<$ Resources $>$

Playing video. Web server analyzes the received XML file, and displays it on the page of Web site. The users find the required video resources on website. And the pseudocode of activities after clicking live is as follows.

TriggerChalinel();// triggering channels

$\mathrm{LL}($ );//calling the shortest path and the shortest time algorithm, and selecting nodes for communication.

LF();//calling the least data block owner downloading algorithm, selecting the data for download. sendData()// the received data is sent to player for playing.

\{

if(sendHead)sendRawData();// If it is video file head, the data is sent to the player. if(senoata)sendRawData();//If it is video data, the data is sent to player

\}

\section{Conclusions}

The Paper applies P2P streaming media system to network teaching. The generated system is tested in the machine room. The users can accurately receive the video information. With the increase of watching time, there is the problem of waiting for buffering, the reason for which is that the occupied user memory is not released timely in implementing programming. 


\section{References}

[1] Padmanabhan, V N., et al., Distributing streaming media content using cooperative networking. Proceedings of the 12th international workshop on Network and operating systems support for digital audio and video, 2002:177-186.

[2] F. Wu, S.P. Li, Y.Q. Zhang. DCT prediction based progressive fme granularity scalability coding. ICIP 2000, 1998. 556-559.

[3] Y.S. Lin, B.Q. Wang, Z.M. Wang. MixCast: A New Group Communication Model in Large-scale Network.The IEEE 19th International Conference on Advanced Information Networking and Applications.Taipei .2005.307-310.

[4] Yeo CK,etal. A survey of application level multicast techniques. Computer Communications, 2004.27(15):1547-1568.

[5] Banerjee s, Bhattacharjee B, Srinivasan A. Resilient multicast using overlays. IEEE/ACM SIGMETRICS Performance Evaluation Review.2003.102-113.

[6] Chu, Y, et al., Enabling conferencing applications on the Internet using overlay muilticast architecture. Proceedings of the 2001 SIGCOMM conference, 2001.31(4):55-67.

[7] Jannotti, J., et al., Overcast: Reliable multicasting with an overlay network. Proceedings of the Fourth Symposium on Operating System Design and Implementation (OSDI), 2000: 197-212. 